\title{
GRAFOS: Uma nova visão da relação de Euler e os Poliedros regulares
}

\author{
GRAPHS: A New View of Euler's Relationship and the \\ Regular Polyhedra
}

\author{
Anna Karla Barros da Trindade ${ }^{1}$ \\ Francisco de Paula Santos de Araujo Junior ${ }^{2}$ \\ Fabiano dos Santos Nascimento ${ }^{3}$
}

\section{RESUMO}

Com o objetivo de transformar o ensino de matemática mais atrativo para alunos professores de matemática da educação básica, foi proposto um estudo mais sofisticado sobre a relação de Euler e os sólidos platônicos, conhecidos também como poliedros de Platão. A relação de Euler sem dúvida é um resultado muito importante na matemática, assim como a demonstração da existência de apenas cinco poliedros regulares. No entanto, esses resultados são bastante previsíveis, em razão da forma que os professores ensinam esse tópico nas escolas, com aulas de geometria desestimulantes aos alunos. Sendo assim, com o objetivo de enriquecer o ensino de geometria no Ensino Médio, buscou-se inserir uma nova forma de demonstrar a relação de Euler e os poliedros regulares, por meio de uma ferramenta pouco conhecida que é a teoria dos grafos. Tais demonstrações não possuem artifícios tão diretos, porém foi necessário introduzir esse estudo com alguns conceitos e exemplos importantes como poliedros, poliedros convexos e não convexos, poliedros regulares, grafo, grau de um vértice, grafos Eulerianos, grafos conexos, grafos planares, isomorfismo entre grafos, grafos regulares e alguns teoremas

1. Mestrado em Matemática - UFPI. Professora de Matemática do Instituto Federal do Piauí IFPI E-mail: anna.trindade @ifpi.edu.br

2. Mestrado em Matemática - UESPI. Professor de Matemática da UESPI. E-mail: pjhatata@hotmail.com

3. Graduado em Lic. Plena em Matemática - UFPI, Especialista em Matemática - FARCE. Professor de Matemática SEMED - Buriti dos Lopes - PI, E-mail: fabianosnascimento2014@hotmail.com 
relevantes. Como resultado desse estudo, foi possível perceber que a relação de Euler e os poliedros regulares podem ser ensinados de maneira simples $e$ interessante com a utilização dos grafos.

Palavras-chave: Poliedros Regulares; Relação de Euler; Grafos. Ensino de Matemática.

\begin{abstract}
In order to make the teaching of Mathematics more attractive to students, and also to Mathematics teachers in Basic Education, a more sophisticated study on the relation of Euler and platonic solids (also known as Plato polyhedra) was proposed. Euler's relation is undoubtedly a very important result in Mathematics as is the demonstration of the existence of only five regular polyhedra. However, these results are quite predictable, as teachers teach this topic in schools, making geometry classes discouraging for students. Thus, from the perspective of enriching the teaching of geometry in high school, we sought to insert a new way of demonstrating the relation of Euler and regular polyhedra, thus introducing a little known tool in high school, graph theory. Such demonstrations do not have such direct devices, but it was necessary to introduce this study with some important concepts and examples such as polyhedra, convex and non-convex polyhedra, regular polyhedra, graph, degree of vertex, Eulerian graphs, connected graphs, planar graphs, isomorphism between graphs, regular graphs and some relevant theorems. As a consequence of this study, it was possible to realize that Euler's relation and regular polyhedra can be taught in a simple and interesting way with the use of graphs.
\end{abstract}

Keywords: Regular Polyhedra; Euler's Relation; Graphs. The Teaching of Mathematics.

\title{
1. INTRODUÇÃO
}

\subsection{Breve nota histórica sobre geometria espacial e o teorema de Euler}

A geometria espacial é uma área da matemática que têm como proposta o estudo das figuras no espaço, ou seja, aquelas que possuem mais de duas dimensões: comprimento, largura e altura. Em geral, a Geometria Espacial pode ser definida como o estudo da geometria no 
espaço. Assim, como Geometria Plana, ela está pautada nos conceitos basilares e intuitivos que chamamos conceitos primitivos, os quais possuem origem na Grécia Antiga e na Mesopotâmia (cerca de 1000 anos a.C). Não obstante, Pitágoras e Platão associavam o estudo da Geometria Espacial ao estudo da Metafísica e da religião; contudo, foi Euclides a se consagrar com sua obra "Elementos", na qual sintetizou os conhecimentos acerca do tema até os seus dias. Entretanto, os estudos de Geometria Espacial permaneceram estanques até o fim da Idade Média, quando Leonardo Fibonacci (1170-1240) escreve a "Practica Geometriae" e, séculos depois, Joannes Kepler (1571-1630) rotula o "Steometria" (stereo: volume/metria: medida) o cálculo de volume, em 1615.

Segundo Siqueira (2009), Leonhard Euler (1707-1783) enunciou a relação $\mathrm{V}+\mathrm{F}=\mathrm{A}+2$, onde $\mathrm{V}$ é número de vértices, $\mathrm{A}$ o número de arestas e $\mathrm{F}$ o número de faces de um poliedro, em uma carta a um amigo. Apesar de descobrir esta relação, Euler não tinha uma definição formal de poliedros.

Apenas em 1893, Henri Poincaré (1854-1912) esclareceu o verdadeiro significado da relação $\mathrm{V}-\mathrm{A}+\mathrm{F}=2$ e estendeu seu conceito. A partir dos estudos de Poincaré, o número $\mathrm{V}-\mathrm{A}+\mathrm{F}$ se tornou muito importante na Matemática com aplicações em Topologia, Análise, Geometria Diferencial e Equações Diferenciais (LIMA, 1985).

O teorema de Euler é um teorema conhecido da geometria espacial, visto por sua vez no ensino médio. O estudo deste teorema é relevante, pois consta nos Parâmetros Curriculares Nacionais, e busca um estudo sobre a relação entre vértices, arestas e faces de um poliedro convexo.

A estrutura do presente trabalho esta organizado em 6 (seis) seções de essencial importância para o ensino de geometria.

Na seção 1, trata-se de uma breve nota histórica sobre a relação de Euler e a geometria espacial, além de um breve comentário sobre o surgimento dos grafos, que se deu através da tentativa de resolver o problema das pontes de Königsberg.

Na seção 2, trata-se do estudo dos poliedros, especialmente dos poliedros regulares, que são o foco de estudo, além das definições de 
poliedros convexos e não convexos. Neste momento, também serão propostos um estudo sobre as primeiras relações existentes nos poliedros, ressaltando ainda o ensino de matemática na atualidade.

A seção 3 é ponto relevante do trabalho, por se tratar de um estudo introdutório mais aprofundado sobre a teoria dos grafos, considerando a importância de algumas definições e teoremas importantes para o alcance do objetivo geral do trabalho.

$\mathrm{Na}$ seção 4, é realizada uma abordagem inovadora sobre uma nova demonstração da relação de Euler e a existência de apenas cinco poliedros regulares com a utilização de conceitos e resultados básicos de grafos.

$\mathrm{Na}$ seção 5, traz-se a solução do problema das pontes de Königsberg e algumas aplicações no ensino médio que se pode se inserir os grafos como ferramenta de resolução.

E na seção 6, trata-se das considerações finais do presente trabalho, analisando a relevância dos resultados e a importância deste estudo para a inserção de um ensino inovador na educação básica, principalmente no ensino médio.

\subsection{Breve história dos Grafos}

De acordo com Ore (1990), diferente de muitos dos ramos da Matemática que foram motivados por problemas envolvendo cálculos, movimento, entre outros, o desenvolvimento da Teoria de Grafos se deu através de problemas envolvendo jogos e quebra-cabeças, o que do ponto de vista matemático parecia insignificante, mas apesar da aparente trivialidade, cada vez mais chamava a atenção de matemáticos pelos seus resultados teóricos de uma surpreendente variedade e profundidade. Em 1736, o matemático suíço Leonhard Euler (1707-1783) escreveu o primeiro artigo relacionado a grafos, de considerável importância não só para esta teoria como também para a Matemática como um todo. Euler iniciou seus estudos em grafos discutindo um enigma, hoje conhecido como O Problema das Pontes de Königsberg.

A cidade era cortada pelo rio Preguel, que possuía duas ilhas. Como era muito complicado fazer o transporte de cargas e pessoas 
através de barcos, algumas pontes foram construídas para auxiliar neste deslocamento entre as ilhas e as duas margens. Após algum tempo as pessoas começaram a se perguntar se era possível sair de sua casa, passar por cada ponte exatamente uma vez e voltar para a segurança de seu lar. Foi a partir deste questionamento que Euler começou o estudo dos grafos, o qual ele resolveu e determinou um método geral para problemas do mesmo tipo (BIGGS e WILSON, 1998).

Desenvolvimentos recentes na Matemática, particularmente nas suas aplicações, deram grande importância a tal teoria. Já no século XIX, grafos foram usados em circuitos elétricos e diagramas moleculares.

Hoje em dia, além dos grafos aparecerem em campos como a Economia e Biologia, existem tópicos na matemática pura que os utilizam como ferramenta.

\section{O ESTUDO DOS POLIEDROS}

Vamos aqui estudar, de uma forma geral, os sólidos formados por faces, os chamados poliedros. Antes de tudo, é preciso estabelecer uma definição adequada para o nível de estudo que se pretende buscar. Dizer apenas que poliedros são sólidos formados por faces (partes limitadas de um plano), pode dar uma ideia do que eles sejam, mas não serve, absolutamente, como definição. Por isso, vamos recomendar para o estudante do $2^{\circ}$ grau, uma definição, que não permita grandes generalidades, mas seja suficiente para demonstrar os teoremas e propriedades importantes.

Definição 1. Poliedro é uma reunião de um número finito de polígonos planos chamados faces onde:

a) Cada lado de um desses polígonos é também lado de um, e apenas um, outro polígono.

b) A intersecção de duas faces quaisquer, ou é um lado comum, ou é um vértice ou é vazia.

c) É sempre possível ir de um ponto de uma face à um ponto qualquer da outra, sem passar por nenhum vértice (ou seja, cruzando apenas arestas). 
Todo poliedro (no sentido da definição acima), limita uma região do espaço chamada interior desse poliedro. Dizemos que um poliedro é convexo se o seu interior é convexo. Vamos recordar o que isso significa.

"Um conjunto $\mathrm{C}$, do plano ou do espaço, diz-se convexo, quando qualquer segmento de reta que liga os dois pontos de $\mathrm{C}$ está inteiramente contido em C".

No caso dos poliedros, podemos substituir essa definição por outra equivalente, que nos será mais útil:

"Um poliedro é convexo se qualquer reta (não paralela a nenhuma de suas faces) o corta em, no máximo, dois pontos".

E o poliedro não convexo é aquele que tem um ângulo entre duas faces maior que $180^{\circ}$, sendo assim ao se construir uma reta que passe por dois pontos de faces não paralelas, esta corta o poliedro com parte interna e parte externa a ele.

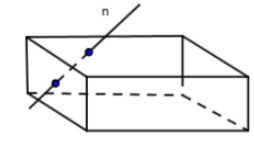

Figura 1. Poliedro convexo

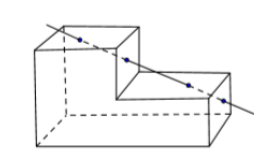

Figura 2. Poliedro não convexo

\subsection{As primeiras relações}

Dado um poliedro, vamos agora tratar do problema de contar as faces, os seus vértices e as suas arestas. Representaremos, então, por $\mathrm{A}$, o número de arestas, por $\mathrm{F}$, o número de faces e por $\mathrm{V}$ o seu número de vértices. Ainda, como as faces podem ser de gêneros diferentes, representaremos por $F_{n}(n \geq 3)$, o número de faces que possuem $n$ lados. Da mesma forma, como os vértices também podem ser de gêneros diferentes, representaremos por $\mathrm{V}_{\mathrm{n}} \mathrm{o}$ número de vértices nos quais concorrem $\mathrm{n}$ arestas, e, observe que, pelo item (b) da definição do poliedro, cada vértice é um ponto comum a três ou mais arestas.

São, então, evidentes as relações:

$$
\begin{aligned}
& F_{n}=F_{3}+F_{4}+\cdots \\
& V_{n}=V_{3}+V_{4}+\cdots
\end{aligned}
$$


Imagine agora que o poliedro foi desmontado e que todas as faces estão em cima de sua mesa. Quantos lados todos esses polígonos possuem? Fácil. Basta multiplicar o número de triângulos por 3 , o número de quadriláteros por 4 , o número de pentágonos por 5 , e assim por diante, e depois somar os resultados. Mas, como cada aresta do poliedro é lado de exatamente duas faces, a soma anterior é igual ao dobro do número de arestas, ou seja,

$$
2 \mathrm{~A}=3 \mathrm{~F}_{3}+4 \mathrm{~F}_{4}+5 \mathrm{~F}_{5}+\cdots
$$

Podemos também contar as arestas observando os vértices do poliedro. Se, em cada vértice, contarmos quantas arestas nele concorre, somando os resultados, obteremos também o dobro do número de arestas (porque cada aresta terá sido contada duas vezes: em um extremo e no outro). Logo,

$$
2 \mathrm{~A}=3 \mathrm{~V}_{3}+4 \mathrm{~V}_{4}+5 \mathrm{~V}_{5}+\cdots
$$

\subsection{O ensino de matemática na atualidade}

O ensino da matemática tem sido alvo de diversas análises, sobretudo no processo de ensino aprendizagem dos alunos atribuídos pelos professores de matemática. Com grandes avanços que se referem à tecnologia, houve a necessidade de trabalhar e adequar novas metodologias e práticas com a vivência dos alunos. Contudo isso, proporcionar aos alunos novas formas de ensino que envolva situações que eles realmente possam adquirir conhecimentos necessários é uma das principais perspectivas que se têm do ensino de matemática nas escolas. No entanto, fazer mudanças não é tão simples, é preciso ter uma preparação e orientação, já que, do contrário, pode-se prejudicar ainda mais o aprendizado, e assim essas mudanças superficiais ou incompletas podem trazer prejuízos educacionais, tanto como ocorre com o ensino tradicional (MICOTTI, 1999).

Atualmente, os alunos apresentam um preocupante desinteresse em aulas de matemática. Isso se deve à ausência de qualidade no ensino. Dessa forma, os alunos se sentem desmotivados a estudar e aprender. De certa forma, os alunos necessitam de uma aprendizagem mais similar e aplicável, ou seja, é importante que os alunos consigam aprender o que é lhes ensinado, de forma que os mesmos adquiram capacidade e possibilidades de aplicar a aprendizagem de tais 
conteúdos com o seu cotidiano de vida, na necessidade de solucionar problemas que os rodeiam. Ou seja, a matemática apresentará utilidades significativas para os alunos. Segundo os PCNS do Ensino Médio (1999, p.251), a matemática é "uma ferramenta que serve para a vida cotidiana e para muitas tarefas específicas em quase todas as atividades humanas". Diante desse pensamento, é necessário que os professores valorizem os planejamentos de suas aulas, ou seja, planejar aulas visando uma dinâmica de associar o conteúdo que está sendo estudado em sala de aula com alguma necessidade do cotidiano, e não apenas propor aulas de reproduções de conteúdos prontos e acabados, conduzindo e iludindo os alunos a decorarem várias fórmulas e teoremas, além de mecanizá-los a realizarem diversos cálculos.

Há questionamentos que tratam o processo de ensino e aprendizagem como algo incerto, inacabado. Ou seja, de quais formas os professores estão estabelecendo os diversos conceitos em sala de aula, como a matemática está sendo ensinada, como os alunos estão lidando com a complexidade dos conteúdos e a ausência de uma praticidade mais adequada para uma compreensão mais flexível. No entanto, é necessário desenvolver uma matemática construtivista, onde os alunos possam construir, pensar, analisar, compreender, refletir, estabelecer relações com o mundo, além de justificar e construir seus próprios conceitos. Para Freire (1996, p. 26) "nas condições de verdadeira aprendizagem os educandos vão se transformando em reais sujeitos da construção e da reconstrução do saber ensinado, ao lado do educador, igualmente sujeito do processo". Evidencia-se também a importância da participação coletiva e individual dos licenciados em matemática, isto é, o professor atua como um agente da sua própria formação.

O professor deve propor aos cursos de sua graduação a suas respectivas análises com o que se diz sobre à importância da sua participação no processo de formação. Ou seja, impor sua liberdade de expressar seus argumentos e formas diferentes que possam ser inseridas no curso de graduação após feitas análises por professores formadores. Dessa maneira, diante deste retrocesso, os professores ganham maior valorização, podendo os mesmos ganhar seu próprio espaço, colaborando, cooperando, participando e fortalecendo tal processo. 


\subsection{Poliedros regulares}

Desde a antiguidade, são conhecidos os poliedros regulares, ou seja, poliedros convexos cujas faces são polígonos regulares iguais e que, em todos os vértices, concorrem o mesmo número de arestas. O livro XIII dos "Elementos" de Euclides (cerca de 100 a.C.) é dedicado inteiramente aos sólidos regulares e contém extensos cálculos que determinam, para cada um, a razão entre o comprimento da aresta e o raio da esfera circunscrita. Na última proposição daquele livro, provase que os poliedros regulares são apenas 5: o tetraedro, o cubo, o octaedro, o dodecaedro e o icosaedro.

Definição 2: Um poliedro convexo é regular quando todas as faces são polígonos regulares iguais e em todos os vértices concorrem o mesmo número de arestas.

O Tetraedro regular é um poliedro representado por uma pirâmide triangular, na qual cada uma das faces laterais são triângulos congruentes ao triângulo da base.

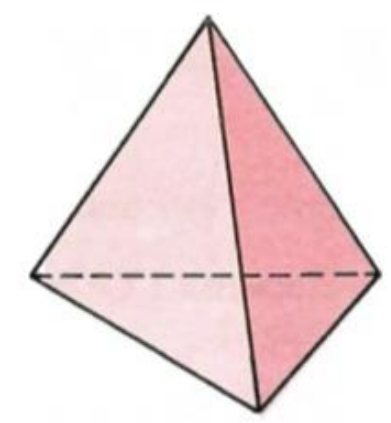

Figura 3. Tetraedro regular

O Hexaedro regular ou cubo é um poliedro regular mais comum, representado por um prisma quadrangular, no qual todas as faces são quadradas. 


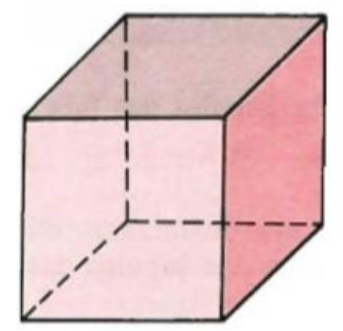

Figura 4. Hexaedro regular

O Octaedro regular é um poliedro regular representado pela fusão de duas pirâmides regulares pelas bases. Neste sólido, todas as faces laterais são triângulos equiláteros.

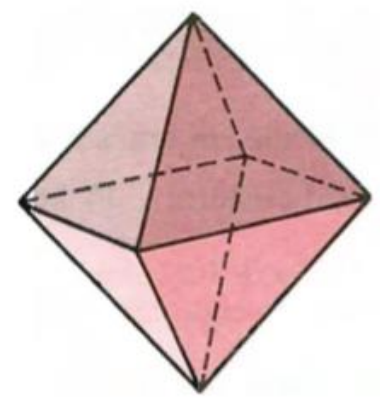

Figura 5. Octaedro regular

O Dodecaedro regular é um poliedro regular que apresenta pentágonos regulares em todas as doze faces.

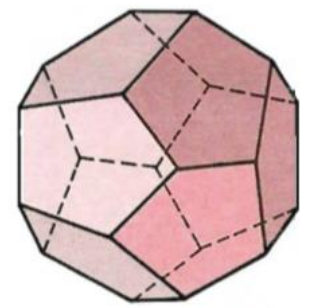

Figura 6. Dodecaedro regular 
O Icosaedro regular é um poliedro regular que apresenta o triângulo equilátero como polígono formador de todas as suas vinte faces.

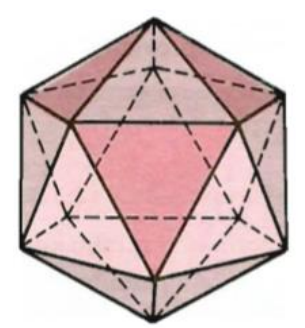

Figura 7. Icosaedro regular

\section{TEORIA DOS GRAFOS}

\subsection{Os grafos}

Desde o século XVIII até nossos dias essa teoria tem conhecido extraordinário desenvolvimento teórico e aplicado. Adotamos então a prática de introduzir alguns temas gerais que dessem uma pequena ideia da variedade de abordagens e problemas que ela pode oferecer. Certamente, muito ficou para depois. O que esperamos é que ao final, o leitor tenha se convencido da utilidade dos conceitos e processos apresentados, mas guardamos o secreto desejo de que os aspectos lúdicos dos grafos o contaminem com o que costumamos chamar de "graphical desease", ou melhor, traduzindo, a febre dos grafos.

Definição 3. Um grafo (finito) $G$ é formado por um par (V (G), $A(G)$ ) onde $V(G)$ é um conjunto finito de vértices e $A(G)$ uma família de pares não ordenados de elementos, chamadas de arestas não necessariamente distintas, de V (G). Uma família é uma coleção de elementos, os quais podem ser repetidos.

O leitor seria capaz de desenhar abaixo sem tirar o lápis do papel? Tem que ir de ponto a ponto e não pode passar pela mesma linha duas vezes. 


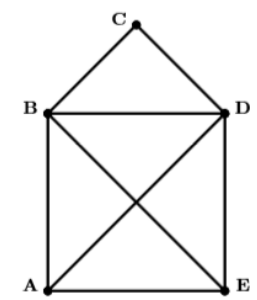

Figura 8. Exemplo de grafo

Foi fácil? Experimente agora começar pelo ponto B.

Bem, esse problema é importante? Pensemos numa pequena cidade com pequeno orçamento. O serviço de recolhimento de lixo é feito por um pequeno caminhão. Queremos evitar o desperdício; uma boa ideia seria fazer o caminhão passar uma única vez por cada rua e retornar ao ponto de partida. Na verdade, é o mesmo problema.

Outro problema que propomos às crianças para que se aquietem é o seguinte: temos que ligar Luz, Gás e Telefone a três casas sem que as linhas se cruzem.

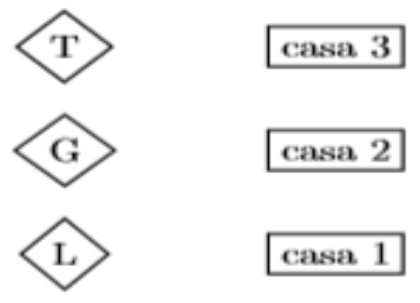

Figura 9. Exemplo

Outra vez, cabe a pergunta: esse problema é importante? Pensemos então numa fábrica de placas de circuito integrado. Encontrar esquemas de ligação que evitem cruzamento é crucial para baratear os custos de manufatura; quanto menos camadas, mais rápido e rentável se torna o serviço. 
Nos dois casos só nos interessou considerar um conjunto de pontos e um conjunto de ligações entre eles. É a essa estrutura que chamamos grafo.

Para que um grafo fique bem definido, temos que ter dois conjuntos:

- O conjunto $\mathrm{V}$, dos vértices.

- $\mathrm{O}$ conjunto $\mathrm{A}$, das arestas.

Em outras palavras, o que nos interessa num grafo é:

\section{- Quem são os vértices.}

Que pares de vértices estão ligados e quais não estão (isto é, quem são as arestas).

\section{Exemplo 1. Grafo Do Campeonato}

Numa escola algumas turmas resolveram realizar um torneio de vôlei. Participam do torneio as turmas $6 \mathrm{~A}, 6 \mathrm{~B}, 7 \mathrm{~A}, 7 \mathrm{~B}, 8 \mathrm{~A}$ e $8 \mathrm{~B}$. Alguns jogos foram realizados até agora: $6 \mathrm{~A}$ jogou com $(7 \mathrm{~A}, 7 \mathrm{~B}, 8 \mathrm{~B})$, 6B jogou com (7A, 8A, 8B), 7A jogou com (6A, 6B), 7B jogou com $(6 \mathrm{~A}, 8 \mathrm{~A}, 8 \mathrm{~B}), 8 \mathrm{~A}$ jogou com $(6 \mathrm{~B}, 7 \mathrm{~B}, 8 \mathrm{~B})$, e $8 \mathrm{~B}$ jogou com $(6 \mathrm{~A}, 6 \mathrm{~B}$, $7 \mathrm{~B}, 8 \mathrm{~A})$.

O exemplo pode não estar correto. Pode ter havido um erro na listagem. Representa-se esta situação através de uma figura. Às turmas serão representadas por pontos e os jogos serão representados por linhas como na Figura 10.

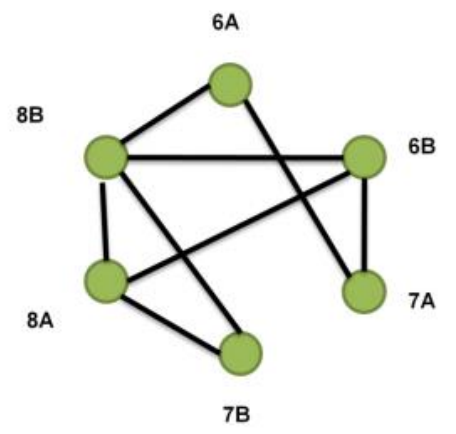

Figura 10. Exemplo de um grafo 


\subsection{Grau do vértice de um grafo}

O grau ou valência de um vértice $\mathrm{v}$, dado por $(\mathrm{gG}(\mathrm{v}))$, em um Grafo é exatamente o número de arestas que incidem naquele vértice $\mathrm{v}$, ressaltando que os laços são contados duas vezes. A Figura 10, exemplifica o grau do vértice do seguinte modo: $(\mathrm{gG}(\mathrm{v} 1))=2$, $(\mathrm{gG}(\mathrm{v} 2))=3,(\mathrm{gG}(\mathrm{v} 3))=4 \mathrm{e}(\mathrm{gG}(\mathrm{v} 4))=3$.

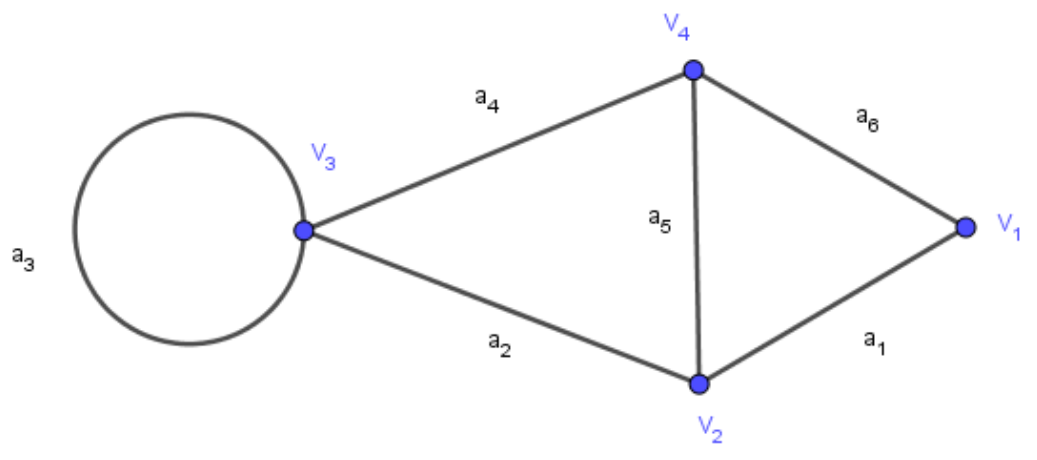

Figura 11. Grau do vértice

Teorema 1. Para todo grafo $G, v \in V(G)$

$$
\sum_{\mathbf{v} \in \mathbf{V}} d(\mathbf{v})=\mathbf{2} \cdot \mathbf{m}
$$

Demonstração: Quando contamos os graus dos vértices estamos contando as extremidades das arestas uma vez. Como cada aresta tem duas extremidades, cada aresta foi contada duas vezes.

Exemplo 2. Da Figura 11, $(\mathrm{gG}(\mathrm{v} 1))=2,(\mathrm{gG}(\mathrm{v} 2))=3,(\mathrm{gG}(\mathrm{v} 3))=4 \mathrm{e}$ $(\mathrm{gG}(\mathrm{v} 4))=3$, assim: $|\mathrm{aG}|=6$. É fácil a verificação do teorema 1 neste exemplo, onde:

$$
\sum_{v \in V} d(v)=2+3+4+3=12
$$

\subsection{Grafo conexo e desconexo}

Um grafo será dito grafo conexo se for possível estabelecer um caminho de qualquer vértice para qualquer outro vértice desse mesmo 
grafo através do percurso das arestas. Caso o Grafo $G$ dado possua pelo menos dois vértices que não estejam ligados através de algum caminho, esse grafo será então um grafo desconexo. Ainda, se houver a possibilidade de estabelecer um caminho de qualquer vértice para qualquer outro vértice, mesmo depois de remover $\mathrm{k}-1$ vértices, diz-se que o grafo está k-conexo.

Exemplo 3. Em um grupo de seis pessoas, Amélia (A), Beatriz (B), Carol (C), Dilma (D), Elizabeth (E) e Fátima (F), sabe-se que:

1) Amélia é irmã de Beatriz, Carol e Fátima;

2) Beatriz é irmã de Carol ;

3) Dilma é irmã de Elizabeth.

Essas pessoas terão relações familiares como irmãs, duas a duas, quando tiverem pelo menos o pai ou a mãe em comum. Alguém de irmãos, temos ainda relações de amizade recíprocas entre essas pessoas do grupo, ou seja, relações em que se uma pessoa $X_{1}$ é amiga da pessoa $X_{2}$, a pessoa $X_{2}$ também será amiga da pessoa $X_{1}$. Acerca dessas relações de amizade, sabe-se que:

i) Amélia conhece Beatriz, Elizabeth e Fátima;

ii) Beatriz conhece Carol;

iii) Carol conhece Dilma, Elizabeth e Fátima;

iv) Dilma conhece Elizabeth.

A Figura 12 representará um grafo com as relações familiares (irmãs) e a Figura 10, um outro grafo para representar as relações de amizade deste grupo.

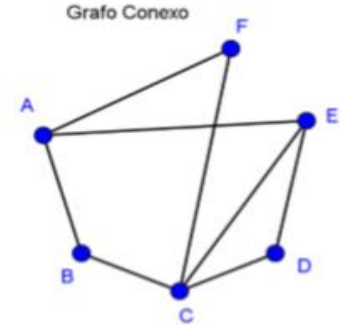

Figura 12. Grafo conexo

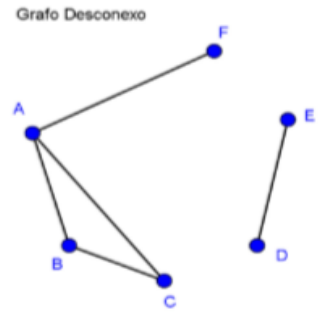

Figura 13. Grafo desconexo 


\subsection{Grafos Eulerianos}

Um grafo com $\mathbf{m}$ arestas é dito euleriano se existe uma trilha fechada de comprimento $\mathbf{m}$ em $\mathrm{G}$; em outras palavras, se podemos percorrer cada aresta uma e só uma vez partindo de um vértice e a ele retornando. Se o grafo não é euleriano, mas tem uma trilha aberta de comprimento $\mathbf{m}$, ele é dito semieuleriano.

Em outras palavras, podemos desenhar um grafo euleriano (ou melhor, uma representação gráfica dele) sem retirar o lápis do papel e retornando ao ponto inicial. Num grafo semieuleriano, começamos num ponto e terminamos em outro.
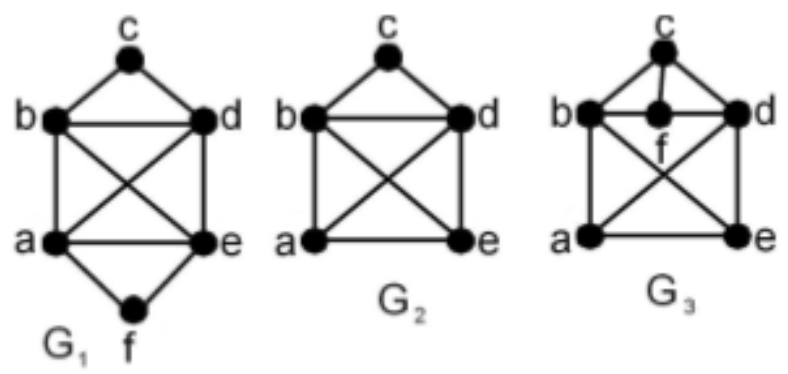

Figura 14. Grafo Euleriano

$\mathrm{Na}$ figura acima, $\mathrm{G}_{1}$ é euleriano (a trilha pode ser a-b-c-d-e-f-a-db-e-a), $G_{2}$ é semieuleriano (a trilha pode ser a-e-b-d-c-b-a-d-e) e $\mathrm{G}_{3}$ não é euleriano, nem semieuleriano.

\subsection{Isomorfismos entre grafos}

Dois grafos $G_{1}$ e $G_{2}$ são ditos isomorfos se existe uma correspondência 1-a-1 entre seus conjuntos de vértices que preserve as adjacências. Ou seja, Dois grafos são ditos isomorfos se houver a possibilidade de se estabelecer uma correspondência biunívoca entre os vértices de um e os vértices do outro e também entre as arestas de um e as arestas do outro, salientando que as arestas devam preservar as suas adjacências. 


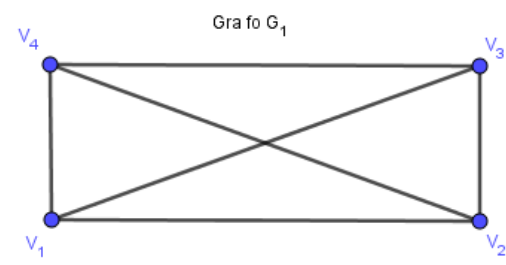

Figura 15. Grafo $G_{1}$

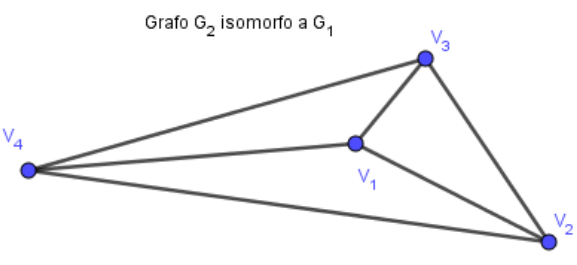

Figura 16. Grafo $\mathrm{G}_{2}$

\subsection{Tipos de grafos}

3.6.1 Grafo nulo ou vazio. Um grafo $G$ é nulo ou vazio quando o conjunto de arestas $\mathrm{A}(\mathrm{G})$ é vazio.

3.6.2 Grafo regular. Um grafo é regular (de grau $\mathrm{k}$, ou ainda $\mathrm{k}$ regular) quando todos os seus vértices têm o mesmo grau (k). A figura 16 mostra um grafo 3-regular, isto é, todos os vértices tem grau 3.

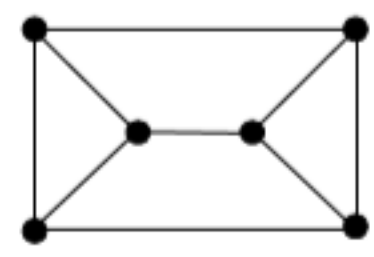

Figura 17. Exemplo de Grafo regular

\subsection{Grafos planares conexos}

Os Grafos planares são aqueles que apresentam uma característica que os tornam diferentes dos demais grafos. Essa diferença é a possibilidade de seu diagrama poder ser representado em um plano sem que duas de suas arestas quaisquer se cruzem. Quando um grafo G aceita uma representação num certo plano $\mathrm{P}$ sem que existam arestas que se interceptam, diz-se que G é um grafo realizável em P. Assim, um grafo diz-se planar se é realizável no plano.

Definição 4. Um Grafo $\mathrm{G}_{1}$ é planar, se existir um certo Grafo $\mathrm{G}_{2}$, isomorfo a $\mathrm{G}_{1}$, cuja representação seja planar. 


\section{RELAÇÃO DE EULER: Uma nova demonstração}

Sobre os Sólidos Platônicos mencionados anteriormente, usou-se a relação de Euler para poliedros, dada por: $\mathrm{V}-\mathrm{A}+\mathrm{F}=2$, onde $\mathrm{V}$ é o número de vértices de um poliedro convexo, A é o número de arestas e F é o número de faces.

Nota-se que a relação de Euler para poliedros é válida também para grafos planares conexos. Afinal, existe uma forma elementar de levar uma instância do primeiro problema para uma instância do segundo: sobre uma face do poliedro, planifique o poliedro de modo que todas as faces estejam sobre um mesmo plano, daí iremos provar a relação para grafos planares conexos.

Dado um grafo planar, isto é, um grafo desenhado sobre o plano e sem intersecções entre arestas. Chamando de faces internas aquelas regiões limitadas pelas arestas do grafo que formam um ciclo. A face externa é a única região ilimitada. Considera-se como face tanto a interna quanto a externa.

Teorema 2. Para todo poliedro $\mathrm{P}, 3 \leq \rho(\mathrm{P}) \leq 5$, onde $\rho$ é o número de arestas do poliedro.

Teorema 3. Da relação de Euler, se $\mathrm{G}$ é um grafo planar conexo com v vértices, a arestas e $\mathrm{f}$ faces, então vale que $\mathrm{v}-\mathrm{a}+\mathrm{f}=2$.

Demonstração. Por indução em $\mathrm{f}$, prova-se então. Se $\mathrm{f}=1$, então $\mathrm{G}$ é uma árvore e temos que $\mathrm{v}=\mathrm{a}+1$. Daí, a relação é verificada neste caso. Supõe-se que $f>1$ e que para todo grafo conexo com $f-1$ faces a relação seja verdadeira. Como $\mathrm{G}$ contém pelo menos duas faces, $\mathrm{G}$ não é uma árvore, logo $\mathrm{G}$ contém um ciclo. Removendo-se uma aresta deste ciclo, mesmo assim o grafo resultante ainda será conexo e terá o mesmo número de vértices, mas tanto o número de arestas quanto o número de faces diminuirá em uma unidade, o que possibilita aplicar a hipótese de indução, de modo que temos $n-(m-1)-(f-1)=2$. Logo, a relação de Euler vale também para o grafo em seu estado original, como queriamos demonstrar.

Teorema 4. (Classificação dos poliedros regulares). Existem exatamente cinco poliedros regulares.

Demonstração. Sejam P um poliedro regular e G seu grafo planar associado. Considere V, A e F o número de vértices, arestas e faces de 
$\mathrm{P}$, respectivamente. Como as faces de $\mathrm{P}$ são congruentes, cada uma delas é limitada pelo mesmo número $\mathrm{k}$ de arestas. Pelo Teorema 2, temos que $3 \leq \mathrm{k} \leq 5$. Além disso, como o poliedro $\mathrm{P}$ é regular, segue que $\mathrm{G}$ também é regular. Seja $\mathrm{r}$ o grau dos vértices de $\mathrm{G}$, onde $\mathrm{r} \geq 3$. Novamente pelo Teorema 2, temos $r V=2 \mathrm{~A}=\mathrm{kF}$. Agora, pelo Teorema 3, temos:

$$
\begin{aligned}
& 8=4 \mathrm{~V}+4 \mathrm{~F}-4 \mathrm{~A} \\
= & 4 \mathrm{~V}+4 \mathrm{~F}-2 \mathrm{~A}-2 \mathrm{~A} \\
= & 4 \mathrm{~V}+4 \mathrm{~F}-\mathrm{rV}-\mathrm{kF} \\
= & (4-\mathrm{r}) \mathrm{V}+(4-\mathrm{k}) \mathrm{F} .
\end{aligned}
$$

Sabendo que V, A e F são positivos, $3 \leq \mathrm{k} \leq 5$ e $\mathrm{r} \geq 3$ e ainda, como $r$ não possui um limitando superior, devemos determinar os possíveis valores de $\mathrm{r}$ para cada um dos três possíveis valores de $\mathrm{k}$, de modo a valerem as equações

$$
\mathrm{rV}=\mathrm{kF}, \quad 8=(4-\mathrm{r}) \mathrm{V}+(4-\mathrm{k}) \mathrm{F} . \quad \text { Equação }(\mathbf{1})
$$

Tomando $\mathrm{k}=3$ na Equação (1), obtemos $\mathrm{rV}=3 \mathrm{~F}$ e $8=(4-\mathrm{r}) \mathrm{V}+\mathrm{F}$. Substituindo $\mathrm{F}=\frac{r V}{3}$ na segunda equação,

$8=(4-\mathrm{r}) \mathrm{V}+\frac{r V}{3} \Rightarrow 24=12 \mathrm{~V}-3 \mathrm{rV}+\mathrm{rV} \Rightarrow 24=(12-2 \mathrm{r}) \mathrm{V} \Rightarrow \mathrm{V}=$ $\frac{24}{12-2 r} \Rightarrow \mathrm{V}=\frac{12}{6-r}$

Os possíveis divisores de 12 são $1,2,3,4,6$ e 12 ,e, portanto os possíveis valores para $\mathrm{r}$ são $5,4,3,2,0 \mathrm{e}-6$. Mas como $\mathrm{r} \geq 3$, devemos ter $\mathrm{r}=3,4,5$. Fazendo a mesma análise para $\mathrm{k}=4 \mathrm{e} \mathrm{k}=5$, obtemos $r=3$ em ambos os casos. Para cada par $(k, r)$ determinados acima, obtemos os possíveis valores para $\mathrm{V}, \mathrm{A}, \mathrm{F}$ por meio das Equações (1). Para $\mathrm{k}=3$ e $\mathrm{r}=3$, as equações $\mathrm{V}=\mathrm{F}$ e $8=\mathrm{V}+\mathrm{F}$ fornecem $\mathrm{V}=\mathrm{F}=4$, ou seja, um tetraedro.

Para $\mathrm{k}=3$ e $\mathrm{r}=4$, as equações $4 \mathrm{~V}=3 \mathrm{~F}$ e $8=\mathrm{F}$ fornecem $\mathrm{V}=6$, ou seja, um octaedro. Para $\mathrm{k}=3 \mathrm{e} \mathrm{r}=5$, as equações $5 \mathrm{~V}=3 \mathrm{~F}$ e $8=-\mathrm{V}$ $+\mathrm{F}$ fornecem $\mathrm{V}=12$ e $\mathrm{F}=20$, ou seja, um icosaedro. Para $\mathrm{k}=4 \mathrm{e} \mathrm{r}=$ 3 , as equações $3 \mathrm{~V}=4 \mathrm{~F}$ e $8=\mathrm{V}$ fornecem $\mathrm{F}=6$, ou seja, um cubo. 
Para $\mathrm{k}=5$ e $\mathrm{r}=3$, as equações $3 \mathrm{~V}=5 \mathrm{~F}$ e $8=\mathrm{V}-\mathrm{F}$ fornecem $\mathrm{F}=$ $12 \mathrm{~V}=20$, ou seja, um dodecaedro.

Portanto, existem apenas 5 poliedros regulares.

\section{GRAFOS: Algumas aplicações no ensino médio}

\subsection{O problema das pontes Königsberg}

Inicia-se abordando a mais antiga citação sobre essa teoria, que ocorreu no ano de 1736, protagonizada pelo matemático suíço Leonhard Euler em seu artigo "The Seven Bridges of Konigsberg". Este problema foi o precursor, cuja solução envolveu conceitos do que viria a ser a Teoria dos Grafos e ficou conhecido como "Problema das Pontes de Königsberg".

Este problema consistia em ver a possibilidade de percorrer todas as pontes que ligavam as quatro regiões separadas pelo Rio Preguel, sem passar pela mesma ponte mais de uma vez. No mesmo ano, Euler analisou o problema trocando as regiões por vértices e as pontes por arestas, modelando o problema, e desta forma provou que não existia solução para o problema das pontes de Königsberg.

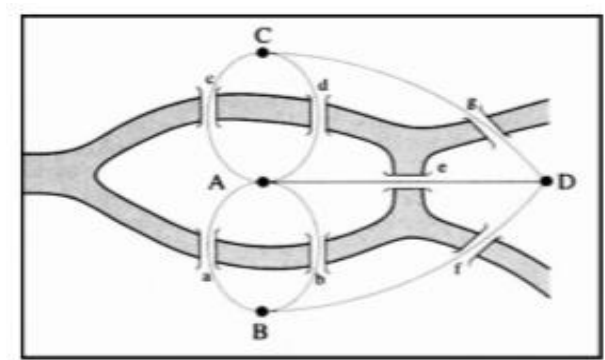

Figura 18.

Primeiro grafo da história

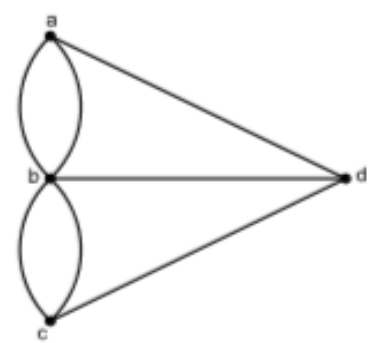

Figura 19.

Grafo das Pontes de Königsberg

Utilizando algo muito simples, que não existia na época de Euler e que formava um desenho no qual tinha alguns pontos e linhas ligando pares de pontos, o então grafo. No problema das pontes, o grafo de Euler tinha quatro regiões: um representando uma das margens do rio, 
o segundo a outra margem; o terceiro ponto representava uma das ilhas e o quarto outra ilha. Sendo assim, o grafo tinha quatro pontos (vértices) e sete linhas (arestas), que representavam as pontes. O desafio era fazer um passeio pelo grafo que partisse de um dos quatro vértices, percorresse cada uma das sete arestas uma única vez e voltasse ao ponto (vértice) de partida. Com esta modelagem foi que Euler racionalizou o problema e o resolveu.

Solução - Euler resolveu da seguinte maneira: ao atravessar cada vértice, são gastos exatamente duas arestas, uma para entrar no ponto e outra para sair. Para atravessar qualquer vértice, são gastas duas arestas, uma para entrar no vértice e outra para sair. Ele concluiu que cada vértice deve ter grau par de arestas. Acontece que o grafo das pontes de Königsberg tem pontos de grau ímpar e, portanto, o problema não pode ter solução.

\section{Considerações Finais}

O trabalho mostrou a importância de uma das mais "novas" teorias no campo Matemático, que é a Teoria dos Grafos.

Além de definir o conceito de Grafos de Euler, formalizar uma demonstração da relação de Euller por meio de grafos, e, seguidamente mostra por meio deste artifício a existência de apenas cinco poliedros regulares. $\mathrm{O}$ trabalho contribui significadamente no ensinoaprendizagem desta Teoria na Matemática Básica.

O conceito de Grafo foi tratado pela variedade de aplicações que esta teoria tem para oferecer aos alunos atuais que sempre estão "antenados" com as novas formas de ver o mundo. Os problemas resolvidos construíram uma visão do conceito de como deve ser a matemática e, sobretudo, a geometria através de grafos conectados com a relação de Euler vista para poliedros.

A relevância deste trabalho está na ideia de uma obra que vem contribuir para novas pesquisas e consulta para professores que pesquisem novos métodos em sala de aula e que visem melhorar o ensino da matemática. Acredita-se ainda que esse trabalho venha ser útil aos professores, no incentivo a trabalhar com grafos, quer seja tirada desta proposta ou na preparação do seu próprio material. Esta proposta vem fomentar e despertar um maior interesse nos alunos pela 
Matemática e suas aplicações usando a Teoria dos Grafos.

Este trabalho tornou-se relevante pelo fato de propor uma nova maneira de demonstrar a relação de Euler e a existência dos cinco poliedros de Platão, fazendo que as aulas de matemática sejam mais produtivas e possibilitando para os alunos novas formas de enxergar os problemas de geometria, tanto na escola como na sua vida cotidiana.

Recebido em: 27/11/2019

Aprovado em: 22/04/2020

\section{Referências}

BIGGS, N. L.; LLOYD, E. K.; WILSON, R. J., Graph Theory 17361936. 1. ed.New York: Clarendon Press, 1998.

CAVALCANTE, F. N. S.; SILVA, S. D. S., Grafos e suas aplicações. 2009; Trabalho de Conclusão de Curso (Licenciatura em Matemática) - Centro Universitário Adventista de São Paulo.

COSTA, P. P., Teoria de Grafos e suas Aplicações. 2011; Dissertação (Mestrado em Matemática) - Universidade Estadual Paulista Júlio de Mesquita Filho, Instituto de Geociências e Ciências Exatas.

FREITAS, J. S., O Teorema de Euler para Poliedros e a Topologia dos Grafos no Ensino Básico. 2017; Dissertação (Mestrado Profissional em Matemática) - Universidade Federal do Maranhão, Centro de Ciências Exatas e Tecnologia.

JURKIEWICZ, S., Grafos - uma introdução. OBEMEP, 2009. Disponível em: <http://www.obmep.org.br/docs/apostila5.pdf>. Acesso em: nov. 2019.

JUSTINO, A. P. R., Poliedros de Platão. 2012; Dissertação (Mestrado em Matemática) - Universidade Federal da Paraíba, Centro de Ciências Exatas e da Natureza, departamento de matemática Curso de Licenciatura em Matemática à Distância.

LIMA, E. L., A Característica de Euler Poincaré. Revista Matemática Universitária, $n^{\circ} 1$, p.47-62, 1985.

LOPES, S. A., Métodos Finitos em Matemática. Disponível em: $<$ http://arquivoescolar.org/handle/arquivo-e/45>. Acesso em: nov. 2019. 
MARTINATTO, M. A., Geometria Espacial no Ensino Médio: sugestões de atividades e avaliações para o conteúdo de Prismas e Pirâmides. 2013; Dissertação (Mestrado Profissional em Matemática) - Universidade Federal do Rio Grande, Instituto de Matemática, Estatística e Física.

MIALICH, F. R., Poliedros e Teorema de Euler. 2013; Dissertação (Mestrado Profissional em Matemática) - Universidade Estadual Paulista Júlio de Mesquita Filho, Instituto de Biociências, Letras e Ciências Exatas.

MICOTTI, M. C. O., O ensino e as propostas pedagógicas. In: BICUDO, M. A. V. (org)., Pesquisa em educação matemática: concepções e perspectivas. São Paulo: Editora Unesp, 1999, p. 153167.

ORE, O., Graphs and their Uses. 2. ed. Washington: The Mathematical Associationof America, 1990.

PARÂMETROS Curriculares Nacionais - Ensino Médio. [S.1.], 1999. Disponível em:<http://portal.mec.gov.br〉. Acesso em: nov. 2019.

SILVA, A. S., O Teorema de Euler e algumas aplicações. 2015; Trabalho de Conclusão de Curso (Licenciatura em Matemática) Universidade Estadual da Paraíba, Centro de Ciências e Tecnologia.

SIQUEIRA, R. M., História, Tradição e Pesquisa sob Disputa: O Caso dos Poliedros na Geometria. Revista Brasileira de História da Matemática, Vol.9 nº17, p.53-63, 2009.

MOTA, A. P. Um Estudo Sobre o Teorema de Euler. 2014; Dissertação (Mestrado Profissional em Matemática) - Universidade Federal de Viçosa. 